\title{
New Physics searches at Belle
}

\author{
Jan HASENBUSCH* \\ Uni Bonn \\ E-mail: hasenbusch@physik.uni-bonn.de
}

Rare decays of $B$ mesons into leptonic final states $B \rightarrow \ell v$ are sensitive to New Physics beyond the Standard Model. A new charged current, as for example predicted in two-Higgs-DoubletModels, might lift the helicity suppression of these decays and interfere with the Standard Model weak current at tree level, leading to changes in branchig fractions and momentum spectra. New Physics couplings are predicted to be proportional to the mass of the charged lepton, thus hints for New Physics might be revealed in semileptonic decays to $\tau$ final states, such as $B \rightarrow D^{(*)} \tau \nu$. Studies of leptonic and semileptonic $B$ decays performed by the Belle collaboration are presented. In addition, a novel search for a stable, heavy neutral fermion $X$, produced in $B \rightarrow \ell X$ decays, is presented.

The Belle experiment collected a large dataset of $710 \mathrm{fb}^{-1}$ at the $\Upsilon(4 \mathrm{~S})$ resonance and provides an excellent environment to study such rare decays of $B$ mesons.

The European Physical Society Conference on High Energy Physics

18-24 July, 2013

Stockholm, Sweden

${ }^{*}$ Speaker. 


\section{Introduction}

Leptonic $B$ decays $B \rightarrow \ell v(\ell=e, \mu, \tau)$ are helicity suppressed in the Standard Model (SM). The $B \rightarrow \tau \nu$ decay is the only currently measurable mode due to the large mass of the $\tau$ compared to $e$ and $\mu$. Further suppression, independent of the final state, arises from the small magnitude of the CKM matrix element $V_{u b}$ involved in this process. Due to this suppression in the SM, New Physics (NP) beyond the SM may introduce sizable affects on observables such as branching fractions or momentum spectra. A non-SM charged current can interfere at tree-level with the SM contribution, leading to an increase or decrease of the branching fraction. A possible model is the two-HiggsDoublet-Model (2HDM), predicted in super symmetry, that introduces a charged Higgs-Boson, which can act instead to the $W$ boson.

Semileptonic $B$ decays can be affected in a similar way by NP, resulting in altered branching fractions and lepton polarisation effects. Of great interest are the $B \rightarrow D^{(*)} \tau \nu$ decays, measured to have higher branching fractions than predicted by the SM (cf. Refs. [1, 2]).

The Belle detector [3], located at the KEKB $e^{+} e^{-}$accelerator in Tsukuba, Japan, provides a clean environment to study such rare $B$-decays in $e^{+} e^{-}$collisions. In its ten years of operation Belle recorded data corresponding to an integrated luminosity of $710 \mathrm{fb}^{-1}$ at the $\Upsilon(4 \mathrm{~S})$ resonance. This data sample contains $770 \times 10^{6} B \bar{B}$ pairs.

\section{2. $B \rightarrow \tau \nu$}

The decay $B \rightarrow \tau v$ is less helicity suppressed than the $B$ decays to $e v$ and $\mu \nu$ final states because of the heavy $\tau$ lepton. Nevertheless, the decay of the $\tau$ lepton makes it challenging since in the $\tau$ decay one (hadronic decay) or two (leptonic decay) neutrinos are produced, leading to a final state with up to three neutrinos. There have been several analyses by the Belle $[4,5]$ and $B A B A R$ collaborations $[6,7]$ using hadronic and semileptonic tag methods, i. e. one $B$ in the $B \bar{B}$ event is reconstructed in an hadronic or semileptonic channel. The average branching fraction is $\mathscr{B}(B \rightarrow \tau v)=(1.65 \pm 0.34) \times 10^{-4}[8]$, whereas the SM predicts a lower value of $\left(0.74_{-0.07}^{+0.09}\right) \times$ $10^{-4}[9]$.

One $B$ meson in the event - called $B_{\text {tag }}-$ is fully reconstructed in a hadronic channel by a full reconstruction algorithm. The latest hadronic tag analysis [10] uses the full Belle dataset as well as an improved full reconstruction algorithm, leading to a three times larger sample of tagged $B$ events than in the previous Belle hadronic tag analysis. Due to the known initial conditions at Belle, the charge, flavour and four-momentum of the second $B$ meson $\left(B_{\text {signal }}\right)$ can be inferred from the $B_{\text {tag }}$. The decay of the $B_{\text {signal }}$ can then be analysed separately.

The final states of the $\tau$ decay from the $B_{\text {signal }}$ in the analysis are $\ell v v, \pi v$ and $\rho\left(\pi \pi^{0}\right) \nu$, with $\ell=e, \mu$. Events with additional tacks or neutral pions are rejected. For signal extraction a $2 \mathrm{D}$ binned maximum likelihood fit is performed in $E_{\mathrm{ECL}}$ and $M_{\mathrm{miss}}^{2}$, where $E_{\mathrm{ECL}}$ is the residual energy in the detector after subtracting energy deposits assigned to the $B_{\text {tag }}$ and $B_{\text {signal }}$ decay products and $M_{\text {miss }}^{2}$ is the squared missing four-momentum $p_{\text {miss }}=p_{\text {tot }}-p_{\text {tag }}-p_{\text {sig }}$.

Signal events are expected to have no additional energy deposits whereas background may have larger values of $E_{\mathrm{ECL}}$, e. g. from non reconstructed neutral pions. 
For the $\tau$ decays to leptonic final states high values of $M_{\text {miss }}^{2}$ are expected due to the three neutrinos, while the hadronic final states have smaller values. Background events are found to have intermediate $M_{\text {miss }}^{2}$ values because, after passing the requirement of a single track on the signal side, these events typically mimic missing mass due to wrongly reconstructed or missing tracks. To pass the single track requirement, these events typically have missing tracks which result in missing momentum. The missing mass is found to have a small correlation with $E_{\mathrm{ECL}}$. Both distributions are shown in Fig. 1. A veto against long-lived neutral kaons is applied, which increases the signal
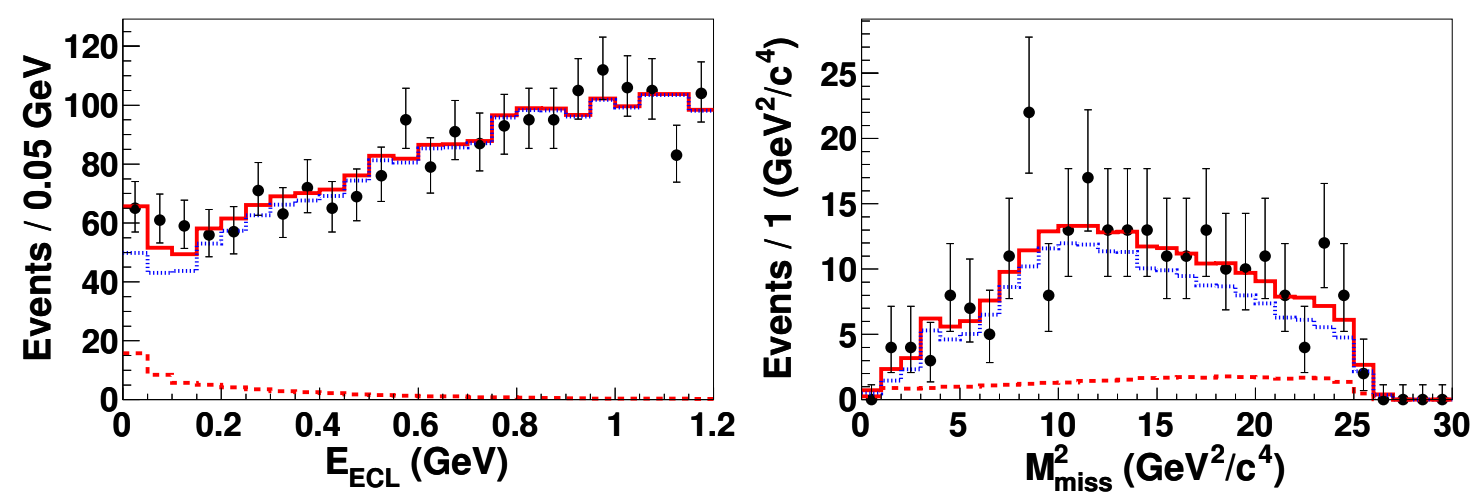

Figure 1: Projections obtained in the maximum likelihood fit in $E_{\mathrm{ECL}}$ (left) and $M_{\mathrm{miss}}^{2}$ (right, for $E_{\mathrm{ECL}}<$ $0.2 \mathrm{GeV}$ ). Data points are shown in black with error bars, the dotted blue line is the sum of all backgrounds and red is the signal. Shown is the sum of all considered final states.

sensitivity by $5 \%$.

The efficiency and measured signal yield is $1.12 \times 10^{-3}$ and $62_{-22}^{+23}$ (stat) \pm 6 (sys) events, respectively, corresponding to a branching fraction of

$$
\mathscr{B}(B \rightarrow \tau v)=\left(0.72_{-0.25}^{+0.27}(\text { stat }) \pm 0.11(\text { sys })\right) \times 10^{-4}
$$

This result is compatible with the prediction by the SM and reduces the world average value to $\mathscr{B}(B \rightarrow \tau v)=(1.14 \pm 0.22) \times 10^{-4}$ [8], i.e. lowers the tension between SM prediction and the world average value to less than $2 \sigma$. An overview of the latest Belle hadronic tag result and previous results is given in Fig. 2.

Separate results for the $e, \mu, \pi$ and $\rho$ subdecay modes are consistent with each other within one standard deviaton. The main systematic uncertainties arise from the $K_{L}$ veto and the description of the background probability density functions (PDF).

\section{3. $B \rightarrow \mu \nu$ and $B \rightarrow e v$}

The highly suppressed $B \rightarrow \mu v$ and $B \rightarrow e v$ final states are predicted to have SM branching fractions of $\mathscr{O}\left(10^{-7}\right)$ and $\mathscr{O}\left(10^{-11}\right)$ for $\ell=\mu$ and $\ell=e$, respectively. As these decays are twobody decays, the charged lepton momentum in the rest frame of the decaying $B_{\text {signal }}$ meson is $p_{\ell}^{B} \approx \frac{m_{B}}{2}$. This gives a unique signature which can be exploited in this analysis because the $B_{\text {signal }}$ rest frame is known from the hadronic tagging. Most backgrounds are not expected to produce high momentum leptons that can reach the signal region, defined as $2.6 \mathrm{GeV}<p_{\ell}^{\mathrm{B}}<2.7 \mathrm{GeV}$. 


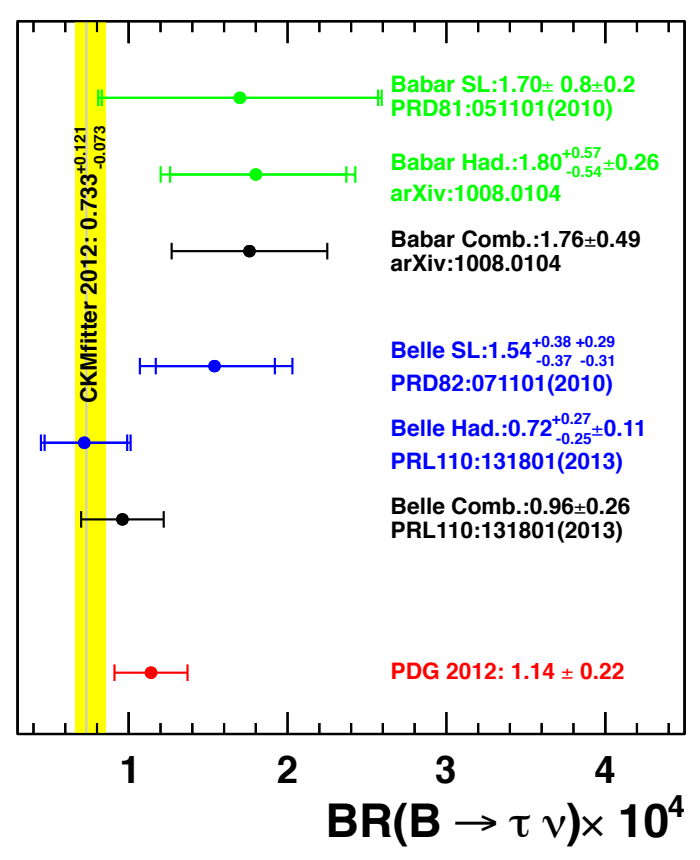

Figure 2: $B \rightarrow \tau \nu$ branching fraction results from Belle and $B A B A R$ with hadronic and semileptonic tagging. Shown is the prediction by the CKM fitter group [9] as well as the current world average.

The exception is the $e^{+} e^{-} \rightarrow q \bar{q}(q=u, d, s, c)$ continuum background which may contain high momentum leptons or misidentified lepton candidates. These events are suppressed by a selection on $\cos \left(\theta_{\text {thrust }}\right)$, where $\theta_{\text {thrust }}$ is defined as the angle of the thrust of the $B_{\text {tag }}$ and the charged lepton momentum.

The charged signal lepton is required to have a lab frame momentum $p_{\ell}^{\text {lab }}>1.8 \mathrm{GeV}$ and originates from close to the interaction point. Events with $E_{\mathrm{ECL}}>0.5 \mathrm{GeV}$ are rejected.

The contamination of the signal region with background events is estimated from Monte Carlo (MC) simulations. For the various background components the shape of the charged lepton momentum spectrum is parametrised by smooth probability density functions (PDF) using unbinned maximum likelihood fits to the MC prediction. The overall background is then obtained in a sideband region of lower lepton momentum $2.0 \mathrm{GeV}<p_{\ell}^{\mathrm{B}}<2.5 \mathrm{GeV}$ and extrapolated into the signal region. The fit result is shown in Fig. 3.

No events are observed in the signal region and $90 \%$ C. L. upper limits on the branching fractions are determined [11]:

$$
\mathscr{B}(B \rightarrow e v)<3.5 \times 10^{-6}
$$

and

$$
\mathscr{B}(B \rightarrow \mu v)<2.5 \times 10^{-6} .
$$

These are the most stringent limits on $B \rightarrow \ell v$ decays using a hadronic tag method. Previous results from Belle and BABAR using a loose tagging method (i. e. tracks and photons excluding the signal lepton have to be compatible with the recoiling $B$ meson) are $\mathscr{B}(B \rightarrow e v)<0.98 \times 10^{-6}$ [12] and $\mathscr{B}(B \rightarrow \mu v)<1.0 \times 10^{-6}$ [13], respectively. 

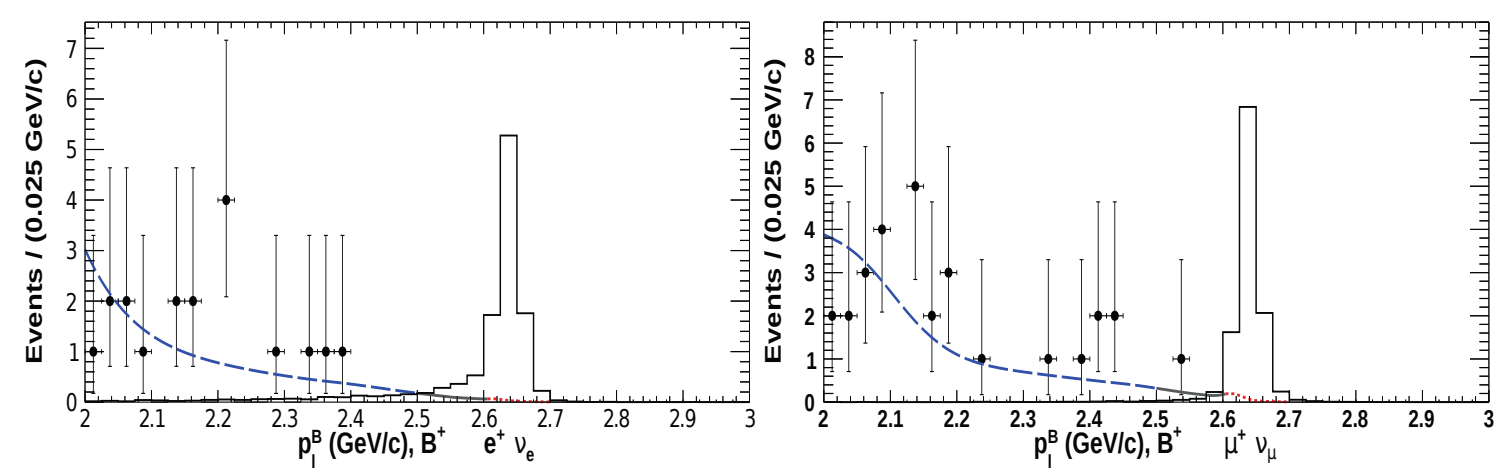

Figure 3: Results of the fit to the $p_{\ell}^{\mathrm{B}}$ spectrum for $B \rightarrow e v$ (left) and $B \rightarrow \mu v$ (right) decays. Data points are shown in black with error bars. The black histogram shows the expected signal shape with arbitrary normalisation. The sum of PDFs is shown as a dashed blue line in the sideband region $\left(2.0 \mathrm{GeV}<p_{\ell}^{\mathrm{B}}<\right.$ $2.5 \mathrm{GeV})$, where the normalisation was obtained. In the signal region $\left(2.6 \mathrm{GeV}<p_{\ell}^{\mathrm{B}}<2.7 \mathrm{GeV}\right)$ the sum of PDFs is shown as a dotted red line.

4. $B \rightarrow \ell X$

A search for a new, heavy neutral fermion $X$ that is stable or long-lived and thus decays outside the detector is performed. This Belle analysis is sensitive to masses from $m_{X}=1.0 \mathrm{GeV}$ up to $1.8 \mathrm{GeV}$. The mass range is chosen such because above $m_{X}=1.8 \mathrm{GeV}$ the amount of background becomes large whereas $m_{X}=1.0 \mathrm{GeV}$ is chosen to distinguish signal from $B \rightarrow \ell v$ decays. No additional assumptions about $X$ are made at this point.

The analysis is performed in a similar way as the $B \rightarrow \ell v$ analysis. The selection criteria are loosened compared to $B \rightarrow \ell v$, i. e. the lepton momentum in the lab frame is $p_{\ell}^{\mathrm{lab}}>1 \mathrm{GeV}$ and there is a looser requirement on the lepton impact parameter as well. The momentum of the charged lepton is known in the $B_{\text {signal }}$ rest frame, but it depends on $m_{X}$. For every step in $m_{X}$ the signal region is chosen such that it minimises the expected upper limit, determined with Toy-MC studies. The backgrounds are modelled with PDFs obtained from fits to MC distributions, as described for $B \rightarrow \ell v$.

The resulting upper limits on $\mathscr{B}(B \rightarrow \ell X)$ are at the order of $10^{-6}$ at $90 \%$ C. L. and are shown in Fig. 4. There are several unexpected events in the region of $p_{\ell}^{\mathrm{B}}>1.3 \mathrm{GeV}$ the $\mu X$ final state that increase the upper limit in the $1.4 \mathrm{GeV}<m_{X}<1.7 \mathrm{GeV}$ region. These events have less than $3 \sigma$ significance and thus are most likely a statistical fluctuation.

This search performed by Belle is the first search for a heavy invisible fermion produced in the decay $B \rightarrow \ell X$. No hints for its existence are found and stringent upper limits are set.

\section{5. $B \rightarrow D^{(*)} \tau \nu$}

An excess of $B \rightarrow D^{(*)} \tau \nu$ events above the SM has been found by both, the Belle and BABAR collaborations. Measurements were done with semileptonic and hadronic tagging methods. To reduce theoretical an systematic uncertainties, the ratios $R\left(D^{(*)}\right)=\frac{B \rightarrow D^{(*)} \tau v}{B \rightarrow D^{(*)} \ell v}$ are measured. The published Belle measurements $[14,1]$ are performed using an inclusive tag and extracting the signal in a 2D maximum likelihood fit in tag mass and momentum of the $D$ or $D^{*}$ meson. A pre- 

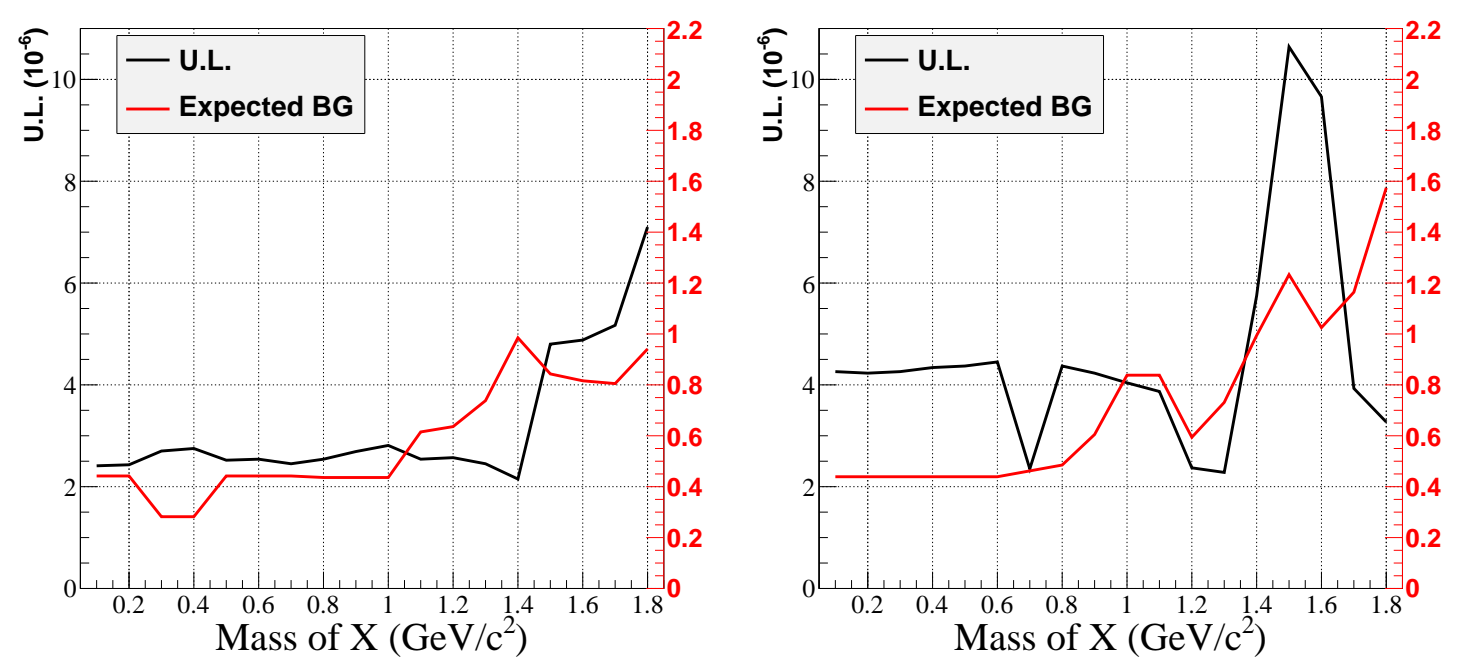

Figure 4: Resulting upper limits on $\mathscr{B}(B \rightarrow \ell X)$ for different masses $m_{X}$, shown as a black line. The increased upper limit in the muon channel around $m_{X}=1.5 \mathrm{GeV}$ is due to unexpected events in the signal region. In addition, the number of expected background events in the signal region is drawn as a red line.

liminary hadronic tag analysis [15] is also available. The signal was extracted in a $2 \mathrm{D}$ fit to the $E_{\mathrm{ECL}}$ and $M_{\text {miss }}^{2}$ distributions. All these Belle measurements use less than $85 \%$ of the full Belle dataset. Looking at charged and neutral $B$ mesons, as well as $D$ and $D^{*}$ separately, each measurement is compatible with the SM within less than $1.5 \sigma$. Taking into account the average of these measurements, as well as the results by $B A B A R$ [7, 2], an excess close to $5 \sigma$ is observed. However, the 2HDM of Type II is not preferred by the Belle measurements. The results for $B \rightarrow D \tau \nu$ and $B \rightarrow D^{*} \tau \nu$ require incompatible values of the ratio of $2 \mathrm{HDM}$ parameters $\frac{\tan \beta}{m_{H}}$. A similar conclusion is drawn from the recent measurement of $B \rightarrow D^{(*)} \tau \nu$ by the BABAR collaboration [2].

A new hadronic tag analysis by Belle is in preparation. The whole Belle dataset and the latest full reconstruction algorithm is used.

\section{Conclusions and outlook}

So far, results from Belle for leptonic $B$ decays do not show evidence for NP contributions. The precise measurement of the $B \rightarrow \tau \nu$ branching fraction is consistent with the SM prediction and decreases the tension between the world average and the predicted value.

For the decays $B \rightarrow \ell v$ with $\ell=e, \mu$ the most stringent upper limits using a hadronic tagging method are obtained. Also measured is the upper limit on the branching fraction of $B \rightarrow \ell X$ with an invisible heavy fermion $X$ in the mass range $1.0 \mathrm{GeV}<m_{X}<1.8 \mathrm{GeV}$. The upper limit is at $\mathscr{O}\left(10^{-} 6\right)$ for this first measurement of that kind.

The semileptonic decay $B \rightarrow D^{(*)} \ell v$ has not yet been measured with the full Belle dataset. A new analysis using hadronic tagging and a signal extraction procedure in two variables will be finished soon. Available measurements show a deviation from the SM when averaged, whereas the single measurements are compatible with the SM prediction. 
If considering a $2 \mathrm{HDM}$ of Type II, regions in the $\tan \beta$ and $m_{H}$ plane are excluded by the result for $B \rightarrow \tau \nu$. In addition to the conclusions drawn from the $B \rightarrow D^{(*)} \tau \nu$ measurements, it is found that the results for $B \rightarrow \tau \nu$, and $B \rightarrow D^{(*)} \tau \nu$ prefer incompatible ratios of $\frac{\tan \beta}{m_{H}}$, too. Thus, the 2HDM Type II is disfavoured by the Belle measurements.

In the future Belle II waits to search for NP with improved detector precision and a fifty times larger data sample to be collected by 2022. An observation of $B \rightarrow \mu v$ is expected within the first recorded $5 \mathrm{ab}^{-1}$ of data [16].

\section{References}

[1] Belle Collaboration, A. Bozek et al., Observation of $B^{+} \rightarrow \bar{D}^{* 0} \tau^{+} v_{\tau}$ and Evidence for $B^{+} \rightarrow \bar{D}^{0} \tau^{+} v_{\tau}$ at Belle, Phys.Rev. D82 (2010) 072005, [arXiv: 1005.2302 ].

[2] BaBar Collaboration, J. Lees et al., Measurement of an Excess of $B \rightarrow D^{(*)} \tau \nu$ Decays and Implications for Charged Higgs Bosons, arXiv:1303.0571.

[3] A. Abashian and et al., The Belle detector, Nuclear Instruments and Methods in Physics Research A 479 (Feb., 2002) 117-232.

[4] Belle Collaboration, K. Ikado et al., Evidence of the Purely Leptonic Decay $B \rightarrow \tau v$, Phys.Rev.Lett. 97 (2006) 251802, [hep-ex/ 0604018 ].

[5] Belle Collaboration, K. Hara et al., Evidence for $B \rightarrow \tau v$ with a semileptonic tagging method, Phys. Rev. 82 (Oct., 2010) 071101, [arXiv: 1006.4201 ].

[6] BaBar Collaboration, P. del Amo Sanchez et al., Evidence for $B^{+} \rightarrow \tau^{+} v_{\tau}$ decays using hadronic $B$ tags, ArXiv e-prints (July, 2010) [arXiv:1008.0104].

[7] Babar Collaboration, B. Aubert et al., A Search for $B^{+} \rightarrow \ell^{+} v_{\ell}$ Recoiling Against $B^{-} \rightarrow D^{0} \ell^{-} \bar{v} X$, Phys.Rev. D81 (2010) 051101, [arXiv: 0912.2453].

[8] Particle Data Group, J. Beringer, et al., Review of particle physics, Phys. Rev. D 86 (Jul, 2012) 010001.

[9] CKMfitter Group, J. Charles et al. Eur. Phys. J. C41 (2005) 1-131, [0 406184 ].

[10] Belle Collaboration, Hara, K. et al., Evidence for $B^{-} \rightarrow \tau^{-} \bar{v}_{\tau}$ with a hadronic tagging method using the full data sample of belle, Phys. Rev. Lett. 110 (Mar, 2013) 131801.

[11] Y. Yook, Leptonic and semileptonic B decays at Belle, PoS ICHEP2012 (2012).

[12] Belle Collaboration, N. Satoyama et al., A search for the rare leptonic decays $B^{+} \rightarrow \mu^{+} v_{\mu}$ and $B^{+} \rightarrow e^{+} v_{e}$, Phys.Lett. B647 (2007) 67-73, [hep-ex/ 0611045$]$.

[13] BaBar Collaboration, B. Aubert et al., Search for the Rare Leptonic Decays $B^{+} \rightarrow l^{+} v_{l}(l=e, \mu)$, Phys.Rev. D79 (2009) 091101, [arXiv: 0903.1220 ].

[14] Belle Collaboration, A. Matyja et al., Observation of $B^{0} \rightarrow D^{*-} \tau^{+} v_{\tau}$ decay at belle, Phys. Rev. Lett. 99 (Nov, 2007) 191807.

[15] Belle Collaboration, I. Adachi et al., Measurement of $B \rightarrow D^{(*)} \tau v$ using full reconstruction tags, arXiv:0910.4301.

[16] Belle II Collaboration, T. Abe, Belle II Technical Design Report, arXiv:1011.0352. 